\title{
Assessment of Rock Mass Quality and Support Estimation along Headrace Tunnel of a Small Hydropower in District Mansehra, Khyber Pakhtunkhwa, Pakistan
}

\author{
Mian Sohail Akram*, Muhammad Zeeshan (), Muhammad Haroon, Kamran Mirza \\ Institute of Geology, University of the Punjab, Lahore, Pakistan \\ Email: *sohail.geo@pu.edu.pk
}

How to cite this paper: Akram, M.S., Zeeshan, M., Haroon, M. and Mirza, K. (2019) Assessment of Rock Mass Quality and Support Estimation along Headrace Tunnel of a Small Hydropower in District Mansehra, Khyber Pakhtunkhwa, Pakistan. Open Journal of Geology, 9, 809-828.

https://doi.org/10.4236/ojg.2019.911092

Received: September 20, 2019

Accepted: October 12, 2019

Published: October 15, 2019

Copyright $\odot 2019$ by author(s) and Scientific Research Publishing Inc. This work is licensed under the Creative Commons Attribution International License (CC BY 4.0).

http://creativecommons.org/licenses/by/4.0/

\section{Open Access}

\begin{abstract}
The main purpose of this study is to classify the rock mass quality by using rock mass quality $(\mathrm{Q})$ and Rock Mass Rating (RMR) systems along headrace tunnel of small hydropower in Mansehra District, Khyber Pakhtunkhwa. Geological field work was carried out to determine the orientation, spacing, aperture, roughness and alteration of discontinuities of rock mass. The quality of rock mass along the tunnel route is classified as good to very poor quality by $\mathrm{Q}$ system, while very good to very poor by RMR classification system. The relatively good rock conditions are acquired via RMR values that are attributed to ground water conditions, joint spacing, RQD and favorable orientation of discontinuities with respect to the tunnel drive. The petrographic studies revealed that study area is mainly comprised of five major geological rock units namely quartz mica schist (QMS), garnet mica schist (GMS), garnet bearing quartz mica schist (G-QMS), calcareous schist (CS), marble (M). The collected samples of quartz mica schist, marble and garnet bearing quartz mica schist are fine to medium grained, compact and are cross cut by few discontinuities having greater spacing. Therefore, these rocks have greater average RQD, Q values, RMR ratings as compared to garnet mica schist and calcareous schist.
\end{abstract}

\section{Keywords}

Tunnel, Petrography, Rock Mass Rating (RMR), Tunneling Quality Index (Q System), Support Estimation

\section{Introduction}

Government of Pakistan is highly committed to curtailing the energy crises of 
the country by developing various hydropower projects especially in northern area of Pakistan. In this regard, a small hydropower project with $2.8 \mathrm{~km}$ long headrace tunnel was proposed in Mansehra district, Khyber Pakhtunkhwa. In many engineering projects including tunneling, ground excavation, foundations and slope stability analysis, geology plays a key role. Among these projects tunnel constructions require a considerable geological input [1]. The overall engineering design weakens by numerous fractures and cracks developed due to tunneling in hard ground conditions leads to deteriorating the physical condition of the ground and rocks. It is necessary to study the geological behavior (spacing and degree of jointing, strength, and attitude) of the strata for safe and economic design of the tunnel [2]. Rock mass classification systems have been effectively applied in many engineering projects especially tunneling projects [3] [4]. To estimate the required support measurements the rock mass classification systems have been proved supportive in assessing the rock mass parameters [5] [6]. Following parameters are used to describe and classify the rock mass into distinct classes:

1) Measurement of degree of jointing known as rock quality designation (RQD)

2) Rock joint parameters such as joint spacing, aperture, orientation, infilling and surface roughness

3) Uniaxial Compressive Strength (UCS) (Strength of intact rock material)

4) Ground water conditions (pressure and flow)

5) In-situ stresses

6) Geological structures such as shear zones, folds and faults

Various researchers [7]-[21] studied the rock mass characterization along tunnel route by using empirical rock mass classification systems e.g. Rock mass rating (RMR), Tunneling quality index ( $\mathrm{Q}$ system) etc. to classify the rock mass with different rock classes according to the physical characteristics of rock mass. This study was conducted to characterize the rock mass along proposed tunnel route by using RMR and Q system. Field studies revealed that study area comprised of five major rock units that have strong impact on the various properties of discontinuities including rock mass strength.

\section{Geology and Rock Mass Characterization}

\subsection{Geological Mapping and Discontinuity Surveys}

The study area lies in the Hazara Nappe that is disjointed from Besham Nappe to the West by dextral Thakot shear zone [22] [23]. The Hazara Nappe dominantly constitutes of metapelites and metapsammites of Pre-Cambrian Tanawal Formation (Figure 1). The Late Cambrian Mansehra porphyritic granite intruded into Tanawal Formation [24]. At some places dolomites of Paleozoic Abbottabad Formation unconformably overlie the Tanawal Formation [25]. Quantitative readings of joints parameters (joint spacing, persistence (length), roughness and joints frequency etc.) were collected at different stations using 


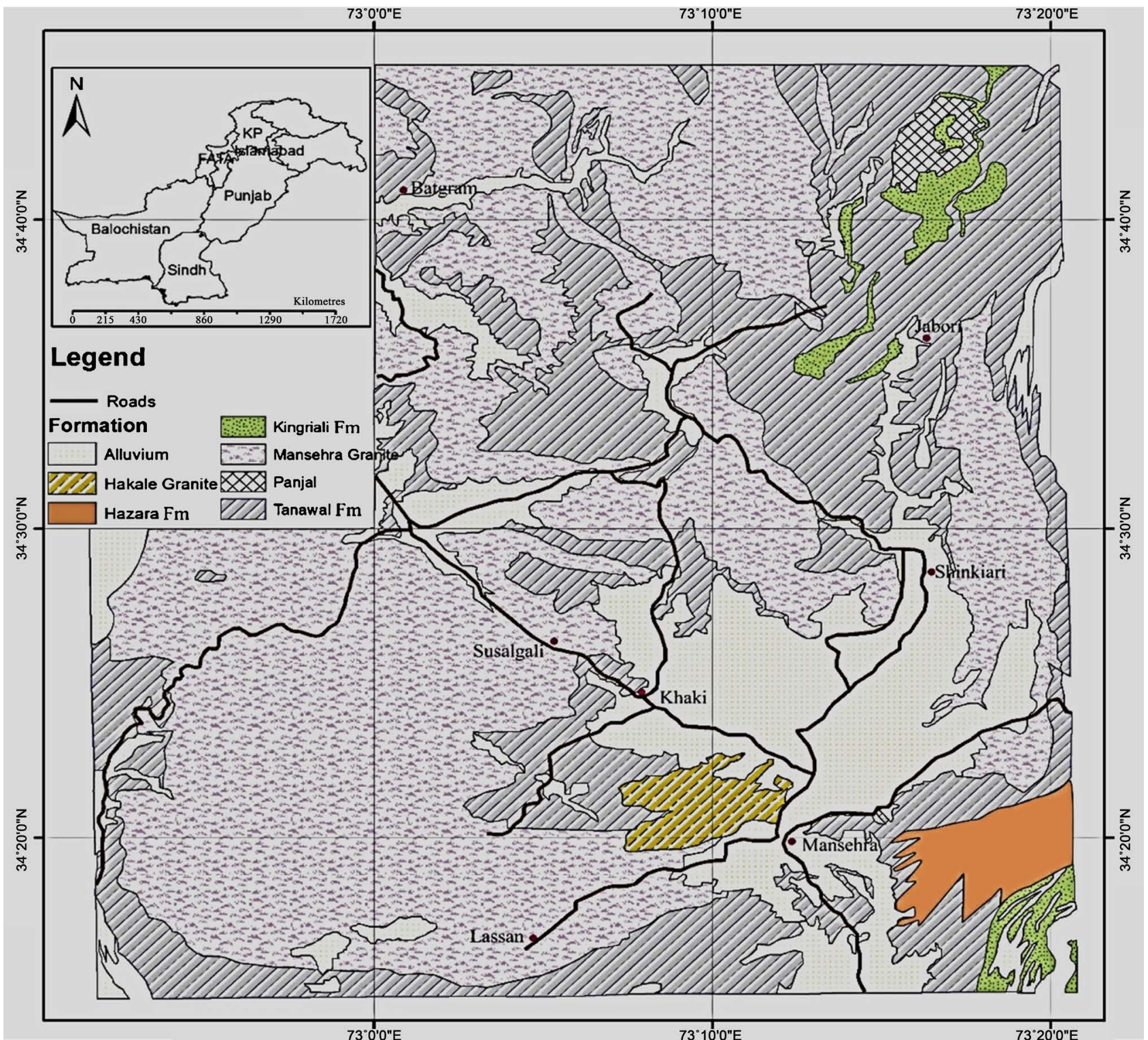

Figure 1. Geological map of Hazara region (Modified after Calkins et al. [22]).

scanline surveys. Geological mapping of tunnel involves the recording of following parameters: geological units, rock type, color, weathering, strength, block size and shape, water sensitivity, discontinuity type, dip direction and dip angle, roughness, spacing, aperture, fill material, persistence, ground water conditions etc.

\subsection{Rock Mass Rating (RMR) and Tunneling Quality Index (Q)}

Rock mass classification systems e.g. tunneling quality index (Q) [26], rock mass rating system (RMR) [2], rock mass index (RMi) [27] and geological strength index (GSI) [28] are used to classify the rock mass and to estimate the support design for underground structures. Rock mass classifications split a specific rock mass into distinct classes of similar manners, but with different qualities from 
one another [5]. Palmstrom [29] Rock mass is the assemblage of rock blocks and fragments divided by discontinuities (joints, bedding planes and faults etc.). A particular value (rating) according to RMR and $\mathrm{Q}$ is given to rock mass according to its behavior to categorize in various rock classes according to the physical properties of discontinuities [5]. The purpose of the classifications is to Bieniawski [2]:

- Ascertain different parameters which affect the rock mass quality

- Divide rock mass into qualitatively different rock classes

- Give guidelines to estimate support for tunnels and mines

Rock structure rating (RSR) by Wickham [4] considered two elements influencing on rock mass behavior: geological parameters and construction parameters. Rock mass rating (RMR) by Bieniawski [2] also known as Geomechanics classification utilizes the basic parameters regarding the geometry and mechanical conditions of the rock mass. Q-system [26] is product of rock mass geometry, interblock shear strength and active stresses encountered during underground excavations. The RMR system was proposed by Bieniawski [2] based on 49 case histories to recognize the stability and support requirements of tunnels. The RMR system has a wide variety of applications in different engineering projects like tunnels, foundations, slopes and mines.

There are six parameters for RMR system [5]:

- Uniaxial Compressive Strength of rock materials (UCS)

- Rock quality designation (RQD)

- Spacing of discontinuities

- Conditions of discontinuities

- Orientation of discontinuities

- Ground water conditions

RMR system classifies strata along tunnel alignment into several zones, each with more or less similar geological features. In most of the circumstances, boundary of these structural regions will overlap with the main geological features such as shear zones, faults and dykes etc. The Q-system consists of six parameters to calculate $\mathrm{Q}$ values by using equation given below and $\mathrm{Q}$ values classify the rock mass into poor and good quality to estimate the required support.

$$
\mathrm{Q}=\mathrm{RQD} / J_{n} \times J_{r} / J_{a} \times J_{w} / \mathrm{SRF}
$$

The parameters are:

RQD = Rock Quality Designation

$J_{n}=$ Joint set number

$J_{r}=$ Joint roughness number

$J_{a}=$ Joint alteration number

$J_{W}=$ Joint water reduction number

$\mathrm{SRF}=$ Stress Reduction Factor

\section{Rock Mass Classification along Tunnel Route}

In this study, all parameters of Q and RMR systems were studied to characterize 
the rock mass along tunnel alignment of $2800 \mathrm{~m}$ length. Total ten (10) scanline surveys were carried out along the tunnel route to record the joints condition. The rock mass was classified in four classes with respect to RMR and Q values (Good to Very Poor rock). The summary of ratings regarding scanline/discontinuity surveys is given in Table 1.

Table 1. Summary of scanline survey executed in the study area.

\begin{tabular}{|c|c|c|c|c|c|c|c|c|c|c|c|}
\hline \multirow[b]{2}{*}{ Location } & \multicolumn{2}{|c|}{ Coordinates } & \multirow{2}{*}{$\begin{array}{c}\text { Discontinuity } \\
\text { Type }\end{array}$} & \multicolumn{2}{|c|}{ Representative Orientation } & \multicolumn{6}{|c|}{ Average Value } \\
\hline & Easting & Northing & & Dip Direction & Dip & $\begin{array}{l}\text { Spacing } \\
(\mathrm{cm})\end{array}$ & $\begin{array}{l}\text { Persistence } \\
\text { (m) }\end{array}$ & $\mathrm{Jr}$ & $\mathrm{Ja}$ & $\mathrm{Jw}$ & SRF \\
\hline \multirow{3}{*}{ Tunnel Intake } & \multirow{3}{*}{295,401} & \multirow{3}{*}{$3,865,172$} & Foliation & 84 & 56 & 12 & 3.5 & 1 & 4 & \multirow{3}{*}{1} & \multirow{3}{*}{2.5} \\
\hline & & & $\mathrm{J}-1$ & 286 & 65 & 27 & 2.7 & 1.5 & 3 & & \\
\hline & & & $\mathrm{J}-2$ & 234 & 60 & 10 & 1.8 & 1 & 3.5 & & \\
\hline \multirow{3}{*}{$\begin{array}{l}\text { Near Nullah } \\
\text { Tunnel Intake }\end{array}$} & \multirow{3}{*}{295,453} & \multirow{3}{*}{$3,865,150$} & Foliation & 81 & 39 & 14 & 2.94 & 2 & 3 & \multirow{3}{*}{1} & \multirow{3}{*}{2} \\
\hline & & & $\mathrm{J}-1$ & 256 & 63 & 15.56 & 1.3 & 2 & 2 & & \\
\hline & & & $\mathrm{J}-2$ & 343 & 76 & 38 & 2 & 1.5 & 3 & & \\
\hline \multirow{4}{*}{$\begin{array}{l}\text { Along Nullah } \\
\text { Downstream } \\
\text { Intake } \\
\text { Structure }\end{array}$} & \multirow{3}{*}{295,545} & \multirow{3}{*}{$3,865,143$} & Foliation & 69 & 58 & 15 & 3.1 & 2 & 2 & \multirow{3}{*}{1} & \multirow{3}{*}{2} \\
\hline & & & $\mathrm{J}-1$ & 307 & 54 & 2.7 & 0.9 & 2 & 2 & & \\
\hline & & & $\mathrm{J}-2$ & 234 & 56 & 1.9 & 2.5 & 2 & 3 & & \\
\hline & \multirow{3}{*}{295,724} & \multirow{3}{*}{$3,865,185$} & Foliation & 347 & 77 & 5.8 & 6 & 1 & 4 & \multirow{3}{*}{1} & \multirow{3}{*}{5.5} \\
\hline \multirow[t]{3}{*}{$\begin{array}{l}\text { Along Tunnel } \\
\text { In Shear Zone }\end{array}$} & & & $J-1$ & 70 & 50 & 8.2 & 0.77 & 1 & 3 & & \\
\hline & & & $\mathrm{J}-2$ & 210 & 42 & 8.6 & 0.53 & 1 & 4 & & \\
\hline & \multirow{3}{*}{296,910} & \multirow{3}{*}{$3,863,742$} & Foliation & 249 & 61 & 10 & 8 & 1.5 & 3 & & \\
\hline \multirow[t]{2}{*}{$\begin{array}{c}\text { Tunnel } \\
\text { Alignment }\end{array}$} & & & $J-1$ & 57 & 58 & 4.5 & 0.5 & 1.5 & 3 & 1 & 2.5 \\
\hline & & & $\mathrm{J}-2$ & 142 & 56 & 2 & 1.5 & 1 & 4 & & \\
\hline & & & Foliation & 258 & 77 & 12.6 & 18.09 & 3 & 2 & & \\
\hline Along Tunnel & 297,307 & $3,863,476$ & $\mathrm{~J}-1$ & 111 & 21 & 15.69 & 3.38 & 3 & 3 & 1 & 2.5 \\
\hline & & & $\mathrm{J}-2$ & 163 & 83 & 50.9 & 9.07 & 2 & 2 & & \\
\hline & & & Foliation & 253 & 60 & 9.1 & 8 & 1 & 3 & & \\
\hline $\begin{array}{c}\text { Near } \\
\text { Tunnel Outlet }\end{array}$ & 297,365 & $3,863,256$ & $\mathrm{~J}-1$ & 131 & 59 & 2.6 & 1.5 & 1 & 3 & 1 & 2.5 \\
\hline & & & $\mathrm{J}-2$ & 354 & 57 & 0.4 & 0.9 & 1.5 & 4 & & \\
\hline Tunnel Outlet & 207570 & 2062201 & Foliation & 264 & 87 & 11.28 & 2.5 & 3 & 2 & & \\
\hline 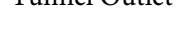 & 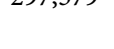 & 250 & $J-1$ & 190 & 87 & 25.4 & 1.4 & 3 & 2 & 1 & 2 \\
\hline Along the & & & Foliation & 77 & 78 & 64.5 & 18.6 & 2 & 3 & & \\
\hline Nullah & 297,962 & $3,863,277$ & $\mathrm{~J}-1$ & 207 & 73 & 40.28 & 12.95 & 3 & 3 & 1 & 2 \\
\hline Downstream & & & $\mathrm{J}-2$ & 47 & 33 & 123.5 & 6.15 & 2 & 2 & & \\
\hline & & & Foliation & 227 & 73 & 17.18 & 2.04 & 2 & 4 & & \\
\hline $\begin{array}{l}\text { Along Road } \\
\text { Near Bridge }\end{array}$ & 297,952 & $3,863,038$ & $J-1$ & 213 & 39 & 25.12 & 1.41 & 1 & 3 & 1 & 2.5 \\
\hline & & & $J-2$ & 350 & 74 & 19.18 & 1.45 & 2 & 3 & & \\
\hline
\end{tabular}




\subsection{Tunnelling Quality Index (Q) of Study Area}

The joint set number $\left(J_{n}\right)$, roughness $\left(J_{r}\right)$, alteration $\left(J_{a}\right)$, water reduction $\left(J_{w}\right)$ and Stress Reduction Factor (SRF) values were assessed during scanline surveys. The $\mathrm{Q}$ value varies between 0.01 and 13.33. The rock mass classified as good (B) to very poor (E) rocks. The rock type, rock mass parameters, calculated $\mathrm{Q}$ values and rock class are given in Table 2.

Table 2. Calculated Q-values and rock class along tunnel alignment.

\begin{tabular}{|c|c|c|c|c|c|c|}
\hline Station (m) & Rock Type & $\mathrm{RQD} / J_{n}$ & $J_{r} / J_{a}$ & $J_{w} / \mathrm{SRF}$ & Q-Value & Rock Class \\
\hline 10 & QMS & 4.17 & 1.00 & 0.20 & 0.83 & Very Poor (E) \\
\hline 25 & GMS & 4.17 & 1.00 & 0.40 & 1.67 & Poor (D) \\
\hline 40 & QMS & 4.00 & 1.00 & 0.40 & 1.60 & \\
\hline 50 & & 3.75 & 1.00 & 0.40 & 1.50 & \\
\hline 60 & GMS & 4.44 & 1.00 & 0.26 & 1.17 & \\
\hline 67 & & 3.89 & 1.50 & 0.40 & 2.33 & \\
\hline 75 & & 3.89 & 1.50 & 0.26 & 1.54 & \\
\hline 80 & & 4.44 & 1.50 & 0.26 & 1.76 & \\
\hline 90 & CS & 3.33 & 1.00 & 0.26 & 0.88 & Very Poor (E) \\
\hline 100 & & 3.33 & 1.50 & 0.20 & 1.00 & Poor (D) \\
\hline 105 & GMS & 4.44 & 1.00 & 0.26 & 1.17 & \\
\hline 120 & & 3.89 & 1.33 & 0.40 & 2.07 & \\
\hline 135 & & 3.89 & 1.00 & 0.20 & 0.78 & Very Poor (E) \\
\hline 145 & & 4.44 & 1.33 & 0.33 & 1.96 & Poor (D) \\
\hline 160 & $\mathrm{QT}+\mathrm{QMS}$ & 4.44 & 1.33 & 0.50 & 2.96 & \\
\hline 175 & & 3.33 & 1.33 & 0.26 & 1.17 & \\
\hline 183 & GMS & 2.50 & 1.33 & 0.50 & 1.67 & \\
\hline 190 & & 2.92 & 1.50 & 0.20 & 0.88 & Very Poor (E) \\
\hline 200 & GMS + SCH_M & 3.89 & 1.50 & 0.20 & 1.17 & Poor (D) \\
\hline 210 & & 2.50 & 1.00 & 0.40 & 1.00 & \\
\hline 225 & & 4.44 & 1.00 & 0.50 & 2.22 & \\
\hline 230 & & 5.00 & 1.00 & 0.50 & 2.50 & \\
\hline 250 & $\mathrm{GMS}+\mathrm{M}$ & 5.00 & 1.50 & 0.40 & 3.00 & \\
\hline 260 & GMS & 3.89 & 1.00 & 0.40 & 1.56 & \\
\hline 275 & & 1.67 & 0.67 & 0.07 & 0.07 & Extremely Poor (F) \\
\hline 286 & & 2.50 & 0.67 & 0.13 & 0.22 & Very Poor (E) \\
\hline 294 & & 1.67 & 0.75 & 0.07 & 0.09 & Extremely Poor (F) \\
\hline 300 & CS & 2.50 & 0.67 & 0.13 & 0.22 & Very Poor (E) \\
\hline 311 & CS & 3.89 & 0.75 & 0.10 & 0.29 & Very Poor (E) \\
\hline 320 & & 5.00 & 1.00 & 0.40 & 2.00 & Poor (D) \\
\hline
\end{tabular}




\section{Continued}

\begin{tabular}{|c|c|c|c|c|c|c|}
\hline 340 & $\mathrm{GMS}+\mathrm{M}$ & 2.22 & 0.67 & 0.20 & 0.30 & Very Poor (E) \\
\hline 350 & & 1.25 & 0.50 & 0.13 & 0.08 & Extremely Poor $(\mathrm{F})$ \\
\hline 370 & & 1.25 & 0.25 & 0.07 & 0.02 & \\
\hline 396 & & 1.25 & 0.50 & 0.13 & 0.08 & \\
\hline 412 & & 2.08 & 0.67 & 0.13 & 0.18 & Very Poor (E) \\
\hline 435 & & 0.83 & 0.25 & 0.07 & 0.01 & Extremely Poor (F) \\
\hline 446 & & 1.67 & 0.67 & 0.20 & 0.22 & Very Poor (E) \\
\hline 460 & $\mathrm{GMS}+\mathrm{QT}$ & 1.25 & 0.25 & 0.13 & 0.04 & Extremely Poor (F) \\
\hline 470 & & 2.08 & 0.67 & 0.20 & 0.28 & Very Poor (E) \\
\hline 522 & GMS & 1.25 & 0.25 & 0.13 & 0.04 & Extremely Poor (F) \\
\hline 527 & & 2.08 & 0.67 & 0.20 & 0.28 & Very Poor (E) \\
\hline 550 & & 1.67 & 0.25 & 0.13 & 0.06 & Extremely Poor (F) \\
\hline 566 & $\mathrm{GMS}+\mathrm{M}$ & 2.08 & 0.67 & 0.13 & 0.19 & Very Poor (E) \\
\hline 600 & $\mathrm{QMS}+\mathrm{M}$ & 1.25 & 0.25 & 0.09 & 0.03 & Extremely Poor $(\mathrm{F})$ \\
\hline 625 & & 7.22 & 1.00 & 0.50 & 3.61 & Poor (D) \\
\hline 638 & & 5.00 & 1.00 & 0.40 & 2.00 & \\
\hline 642 & & 0.83 & 0.20 & 0.14 & 0.02 & Extremely Poor (F) \\
\hline 670 & & 3.75 & 1.50 & 0.40 & 2.25 & Poor (D) \\
\hline 687 & & 6.11 & 1.50 & 0.53 & 4.88 & Fair $(\mathrm{C})$ \\
\hline 692 & $\mathrm{GMS}+\mathrm{M}$ & 4.17 & 1.50 & 0.26 & 1.65 & Poor (D) \\
\hline 696 & & 0.83 & 0.25 & 0.09 & 0.02 & Extremely Poor (F) \\
\hline 720 & & 5.56 & 1.50 & 0.26 & 2.20 & Poor (D) \\
\hline 730 & & 3.89 & 0.67 & 0.09 & 0.23 & Very Poor (E) \\
\hline 741 & & 1.67 & 1.50 & 0.09 & 0.22 & \\
\hline 750 & & 2.50 & 0.25 & 0.09 & 0.06 & Extremely Poor (F) \\
\hline 757 & & 7.78 & 1.00 & 0.40 & 3.11 & Poor (D) \\
\hline 784 & & 7.78 & 1.50 & 0.53 & 6.21 & Fair (C) \\
\hline 800 & $\mathrm{GMS}+\mathrm{M}$ & 6.67 & 1.00 & 0.40 & 2.67 & Poor (D) \\
\hline 812 & $\mathrm{QMS}+\mathrm{M}$ & 7.78 & 1.50 & 0.53 & 6.21 & Fair (C) \\
\hline 825 & & 8.89 & 0.67 & 0.26 & 1.56 & Poor (D) \\
\hline 850 & & 4.58 & 0.67 & 0.40 & 1.22 & \\
\hline 890 & $\mathrm{MB}$ & 6.11 & 1.50 & 0.53 & 4.88 & Fair (C) \\
\hline 900 & $\mathrm{QMS}+\mathrm{M}$ & 5.00 & 1.00 & 0.26 & 1.32 & Poor (D) \\
\hline 907 & MS & 5.00 & 1.00 & 0.40 & 2.00 & \\
\hline 920 & QMS & 3.75 & 0.50 & 0.13 & 0.25 & Very Poor (E) \\
\hline 927 & & 3.75 & 0.50 & 0.09 & 0.17 & Very Poor (E) \\
\hline 960 & & 8.33 & 1.00 & 0.67 & 5.56 & Fair (C) \\
\hline
\end{tabular}




\section{Continued}

\begin{tabular}{|c|c|c|c|c|c|c|}
\hline 975 & & 15.00 & 1.00 & 0.67 & 10.00 & Good (B) \\
\hline 1008 & & 15.83 & 1.00 & 0.50 & 7.92 & Fair (C) \\
\hline 1040 & & 13.33 & 0.67 & 0.40 & 3.56 & Poor (D) \\
\hline 1060 & & 7.78 & 2.00 & 0.50 & 7.78 & Fair (C) \\
\hline 1075 & & 8.89 & 2.00 & 0.33 & 5.87 & \\
\hline 1085 & & 8.33 & 1.50 & 0.50 & 6.25 & \\
\hline 1100 & & 8.33 & 3.00 & 0.33 & 8.25 & \\
\hline 1110 & & 8.89 & 3.00 & 0.50 & 13.33 & Good (B) \\
\hline 1120 & & 8.33 & 3.00 & 0.33 & 8.25 & Fair (C) \\
\hline 1130 & & 8.33 & 1.50 & 0.50 & 6.25 & \\
\hline 1150 & & 8.33 & 0.67 & 0.40 & 2.22 & Poor (D) \\
\hline 1175 & & 5.83 & 0.38 & 0.09 & 0.19 & Very Poor (E) \\
\hline 1200 & & 5.83 & 0.38 & 0.13 & 0.29 & \\
\hline 1225 & CS & 5.67 & 0.25 & 0.13 & 0.19 & \\
\hline 1260 & $\mathrm{CS}+\mathrm{QMS}$ & 6.67 & 0.38 & 0.09 & 0.22 & \\
\hline 1280 & G_QMS + CS & 9.44 & 1.50 & 0.50 & 7.08 & Fair (C) \\
\hline 1290 & & 8.89 & 1.50 & 0.33 & 4.40 & \\
\hline 1315 & G_QMS & 9.44 & 1.50 & 0.50 & 7.08 & \\
\hline 1320 & & 8.89 & 1.50 & 0.33 & 4.40 & \\
\hline 1385 & $\mathrm{MB}+\mathrm{G} \_\mathrm{QMS}+\mathrm{CS}$ & 10.00 & 1.50 & 0.50 & 7.50 & \\
\hline 1425 & $\mathrm{QMS}+\mathrm{G} \_\mathrm{QMS}$ & 8.89 & 0.67 & 0.50 & 2.96 & Poor (D) \\
\hline 1445 & GMS + G_QMS & 10.00 & 0.67 & 0.50 & 3.33 & Poor (D) \\
\hline 1490 & $\begin{array}{c}\mathrm{QMS}+\mathrm{G} \_\mathrm{QMS}+ \\
\mathrm{GMS}\end{array}$ & 10.00 & 1.50 & 0.50 & 7.50 & Fair (C) \\
\hline 1510 & QMS & 7.78 & 0.67 & 0.20 & 1.04 & Poor (D) \\
\hline 1525 & G_QMS + QMS & 6.67 & 0.67 & 0.53 & 2.38 & \\
\hline 1538 & G_QMS & 5.56 & 0.67 & 0.53 & 1.98 & \\
\hline 1549 & & 4.58 & 0.17 & 0.10 & 0.08 & Extremely Poor (F) \\
\hline 1555 & & 5.42 & 0.25 & 0.13 & 0.18 & Very Poor (E) \\
\hline 1586 & & 3.33 & 0.17 & 0.04 & 0.02 & Extremely Poor (F) \\
\hline 1645 & QMS & 2.50 & 0.17 & 0.10 & 0.04 & \\
\hline 1653 & & 2.08 & 0.17 & 0.07 & 0.02 & \\
\hline 1660 & & 2.50 & 0.67 & 0.09 & 0.15 & Very Poor (E) \\
\hline 1690 & & 2.08 & 0.17 & 0.07 & 0.02 & Extremely Poor (F) \\
\hline 1745 & GMS & 4.44 & 0.50 & 0.10 & 0.22 & Very Poor (E) \\
\hline 1760 & & 2.08 & 0.38 & 0.09 & 0.07 & Extremely Poor (F) \\
\hline 1800 & & 3.33 & 0.50 & 0.13 & 0.22 & Very Poor (E) \\
\hline 1808 & QMS & 5.56 & 0.67 & 0.35 & 1.30 & Poor (D) \\
\hline
\end{tabular}




\section{Continued}

\begin{tabular}{|c|c|c|c|c|c|c|}
\hline 1835 & & 5.56 & 0.67 & 0.53 & 1.97 & \\
\hline 1865 & & 5.00 & 1.00 & 0.35 & 1.76 & \\
\hline 1875 & & 5.56 & 0.67 & 0.53 & 1.97 & \\
\hline 1892 & & 5.56 & 0.67 & 0.40 & 1.48 & \\
\hline 1908 & & 3.89 & 0.67 & 0.40 & 1.04 & \\
\hline 1925 & & 8.33 & 0.67 & 0.53 & 2.96 & \\
\hline 1934 & & 8.33 & 0.67 & 0.35 & 1.95 & \\
\hline 1960 & GMS & 8.33 & 0.67 & 0.53 & 2.96 & \\
\hline 1980 & & 9.17 & 0.67 & 0.53 & 3.25 & \\
\hline 2000 & & 9.17 & 0.67 & 0.35 & 2.15 & \\
\hline 2025 & GMS + QMS & 8.33 & 1.00 & 0.35 & 2.93 & \\
\hline 2050 & QMS & 8.33 & 0.67 & 0.35 & 1.95 & \\
\hline 2065 & & 8.33 & 0.67 & 0.53 & 2.96 & \\
\hline 2086 & G_QMS + GMS & 6.67 & 0.67 & 0.35 & 1.56 & \\
\hline 2100 & GMS & 4.44 & 0.67 & 0.40 & 1.19 & Poor (D) \\
\hline 2125 & $\mathrm{QT}+\mathrm{MM}$ & 5.83 & 0.67 & 0.40 & 1.56 & \\
\hline 2141 & MM & 8.33 & 1.00 & 0.40 & 3.33 & \\
\hline 2150 & G_QMS & 6.67 & 1.00 & 0.40 & 2.67 & \\
\hline 2162 & & 6.67 & 1.00 & 0.53 & 3.55 & \\
\hline 2175 & M & 5.83 & 1.00 & 0.40 & 2.33 & \\
\hline 2186 & & 6.67 & 1.00 & 0.53 & 3.55 & \\
\hline 2200 & $\mathrm{QMS}+\mathrm{QT}$ & 6.67 & 0.67 & 0.40 & 1.78 & \\
\hline 2216 & & 7.50 & 0.67 & 0.40 & 2.00 & \\
\hline 2236 & QT & 6.67 & 1.00 & 0.53 & 3.55 & \\
\hline 2252 & & 7.50 & 0.67 & 0.40 & 2.00 & \\
\hline 2265 & & 6.11 & 1.00 & 0.50 & 3.06 & \\
\hline 2275 & & 6.11 & 0.67 & 0.50 & 2.04 & \\
\hline 2292 & & 8.33 & 1.00 & 0.40 & 3.33 & \\
\hline 2352 & & 8.33 & 1.00 & 0.26 & 2.20 & \\
\hline 2317 & & 6.67 & 1.00 & 0.50 & 3.33 & \\
\hline 2330 & & 4.44 & 0.67 & 0.26 & 0.78 & Very Poor (E) \\
\hline 2347 & $\mathrm{QT}+\mathrm{QMS}$ & 5.00 & 0.67 & 0.40 & 1.33 & Poor (D) \\
\hline 2365 & QT & 3.89 & 1.00 & 0.50 & 1.94 & \\
\hline 2376 & $\mathrm{GMS}+\mathrm{M}$ & 3.33 & 0.67 & 0.40 & 0.89 & Very Poor (E) \\
\hline 2393 & $\mathrm{M}+\mathrm{QMS}$ & 4.44 & 0.67 & 0.40 & 1.19 & Poor (D) \\
\hline 2400 & M & 4.44 & 1.00 & 0.50 & 2.22 & \\
\hline 2426 & & 3.33 & 0.67 & 0.50 & 1.11 & \\
\hline
\end{tabular}




\section{Continued}

\begin{tabular}{|c|c|c|c|c|c|c|}
\hline 2440 & & 5.00 & 0.67 & 0.33 & 1.10 & \\
\hline 2455 & & 8.33 & 0.67 & 0.40 & 2.22 & \\
\hline 2468 & & 8.33 & 1.50 & 0.80 & 10.00 & Good (B) \\
\hline 2475 & & 8.33 & 1.50 & 0.53 & 6.60 & Fair (C) \\
\hline 2503 & & 4.44 & 1.50 & 0.80 & 5.33 & \\
\hline 2524 & & 5.56 & 1.50 & 0.80 & 6.67 & \\
\hline 2535 & & 4.44 & 1.50 & 0.80 & 5.33 & \\
\hline 2557 & & 4.44 & 1.50 & 0.50 & 3.33 & Poor (D) \\
\hline 2576 & M & 5.00 & 1.50 & 0.80 & 6.00 & Fair (C) \\
\hline 2587 & & 6.11 & 1.00 & 0.26 & 1.61 & Poor (D) \\
\hline 2594 & & 6.11 & 1.00 & 0.20 & 1.22 & \\
\hline 2603 & & 4.44 & 1.50 & 0.40 & 2.67 & \\
\hline 2610 & & 5.00 & 1.00 & 0.26 & 1.32 & \\
\hline 2624 & G_QMS $+\mathrm{M}$ & 5.00 & 1.00 & 0.40 & 2.00 & \\
\hline 2635 & G_QMS + QMS & 6.67 & 1.00 & 0.26 & 1.76 & \\
\hline 2652 & QMS & 3.33 & 0.38 & 0.20 & 0.25 & Very Poor (E) \\
\hline 2673 & & 6.11 & 1.00 & 0.40 & 2.44 & Poor (D) \\
\hline 2682 & $\mathrm{QMS}+\mathrm{GMS}$ & 4.17 & 1.00 & 0.26 & 1.10 & \\
\hline 2689 & $\mathrm{GMS}+\mathrm{M}$ & 3.33 & 0.50 & 0.20 & 0.33 & Very Poor (E) \\
\hline 2702 & & 4.17 & 1.00 & 0.26 & 1.10 & Poor (D) \\
\hline 2707 & GMS & 4.17 & 1.00 & 0.40 & 1.67 & \\
\hline 2714 & & 7.22 & 1.00 & 0.40 & 2.89 & \\
\hline 2720 & & 6.67 & 1.33 & 0.40 & 3.56 & \\
\hline 2727 & $\mathrm{GMS}+\mathrm{M}$ & 5.56 & 0.67 & 0.40 & 1.48 & \\
\hline 2733 & $\mathrm{MB}+\mathrm{GMS}$ & 6.67 & 1.00 & 0.40 & 2.67 & \\
\hline 2740 & & 5.56 & 0.67 & 0.40 & 1.48 & \\
\hline 2746 & & 5.56 & 1.00 & 0.40 & 2.22 & \\
\hline 2751 & & 7.22 & 1.00 & 0.40 & 2.89 & \\
\hline 2760 & & 6.67 & 1.00 & 0.40 & 2.67 & \\
\hline 2773 & & 6.67 & 1.00 & 0.26 & 1.76 & \\
\hline 2782 & M & 7.22 & 1.00 & 0.40 & 2.89 & \\
\hline 2788 & $\mathrm{MB}+\mathrm{GMS}$ & 7.22 & 1.00 & 0.26 & 1.91 & \\
\hline 2800 & GMS & 6.67 & 1.00 & 0.13 & 0.88 & Very Poor (E) \\
\hline
\end{tabular}

GMS $=$ Garnet Mica Schist, $\mathrm{M}=$ Marble, $\mathrm{MM}=$ Micaceous Marble, $\mathrm{QMS}=$ Quartz Mica Schist, $\mathrm{G}-\mathrm{QMS}=$ Garnet bearing Quartz Mica Schist, CS = Calcareous Schist, $\mathrm{QT}=$ Quartzite, $\mathrm{MS}=$ Micaceous Schist.

\subsection{Rock Mass Rating (RMR) of Study Area}

The parameters including the Uniaxial Compressive Strength (UCS) [30], RQD, 
joint spacing, orientation, persistence, joint surface conditions and ground water conditions were determined to calculate RMR for each rock type section as discussed below and final calculated values of RMR are given in Table 3 with rock quality ranged between very good (I) to very poor (V) rock.

Table 3. Calculated values of RMR and rock class along the tunnel route.

\begin{tabular}{|c|c|c|c|}
\hline Station (m) & Rock Type & RMR-Value & Rock Class \\
\hline 10 & QMS & 38 & Poor rock (IV) \\
\hline 25 & GMS & 43.00 & Fair rock (III) \\
\hline 40 & QMS & 42.00 & \\
\hline 50 & & 42.00 & \\
\hline 60 & GMS & 41.00 & \\
\hline 67 & & 46.00 & \\
\hline 75 & & 43.00 & \\
\hline 80 & & 44.00 & \\
\hline 90 & CS & 38.00 & Poor rock (IV) \\
\hline 100 & & 42.00 & Fair rock (III) \\
\hline 105 & GMS & 41.00 & \\
\hline 120 & & 46.00 & \\
\hline 135 & & 39.00 & Poor rock (IV) \\
\hline 145 & & 44.00 & Fair rock (III) \\
\hline 160 & $\mathrm{QT}+\mathrm{QMS}$ & 55.00 & \\
\hline 175 & & 41.00 & \\
\hline 183 & GMS & 43.00 & \\
\hline 190 & GMS & 38.00 & Poor rock (IV) \\
\hline 200 & $\mathrm{GMS}+\mathrm{SCH} \_\mathrm{M}$ & 41.00 & Fair rock (III) \\
\hline 210 & & 41.00 & \\
\hline 225 & & 47.00 & \\
\hline 230 & & 48.00 & \\
\hline 250 & $\mathrm{GMS}+\mathrm{M}$ & 55.00 & \\
\hline 260 & GMS & 43.00 & \\
\hline 275 & GMS & 16.00 & Very poor rock $(\mathrm{V})$ \\
\hline 286 & & 24.00 & Poor rock (IV) \\
\hline 294 & & 18.00 & Very poor rock $(\mathrm{V})$ \\
\hline 300 & CS & 18.00 & \\
\hline 311 & & 27.00 & Poor rock (IV) \\
\hline 320 & & 23.00 & \\
\hline 340 & $\mathrm{GMS}+\mathrm{M}$ & 24.00 & \\
\hline 350 & & 17.00 & Very poor rock $(\mathrm{V})$ \\
\hline
\end{tabular}




\section{Continued}

\begin{tabular}{|c|c|c|c|}
\hline 370 & & 13.00 & \\
\hline 396 & & 18.00 & \\
\hline 412 & & 22.00 & Poor rock (IV) \\
\hline 435 & & 15.00 & Very poor rock $(\mathrm{V})$ \\
\hline 446 & & 24.00 & Poor rock (IV) \\
\hline 460 & $\mathrm{GMS}+\mathrm{QT}$ & 14.00 & Very poor rock $(\mathrm{V})$ \\
\hline 470 & & 27.00 & Poor rock (IV) \\
\hline 522 & GMS & 13.00 & Very poor rock $(\mathrm{V})$ \\
\hline 527 & & 28.00 & Poor rock (IV) \\
\hline 550 & & 16.00 & Very poor rock $(\mathrm{V})$ \\
\hline 566 & $\mathrm{GMS}+\mathrm{M}$ & 26.00 & Poor rock (IV) \\
\hline 600 & $\mathrm{QMS}+\mathrm{M}$ & 15.00 & Very poor rock $(\mathrm{V})$ \\
\hline 625 & & 58.00 & Fair rock (III) \\
\hline 638 & & 45.00 & \\
\hline 642 & & 12.00 & Very poor rock $(\mathrm{V})$ \\
\hline 670 & & 49.00 & Fair rock (III) \\
\hline 687 & & 86.00 & Very good rock (I) \\
\hline 692 & $\mathrm{GMS}+\mathrm{M}$ & 44.00 & Fair rock (III) \\
\hline 696 & & 13.00 & Very poor rock $(\mathrm{V})$ \\
\hline 720 & & 44.00 & Fair rock (III) \\
\hline 730 & & 26.00 & Poor rock (IV) \\
\hline 741 & $\mathrm{GMS}+\mathrm{M}$ & 27.00 & Poor rock (IV) \\
\hline 750 & & 15.00 & Very poor rock $(\mathrm{V})$ \\
\hline 757 & & 50.00 & Fair rock (III) \\
\hline 784 & & 87.00 & Very good rock (I) \\
\hline 800 & & 46.00 & Fair rock (III) \\
\hline 812 & $\mathrm{QMS}+\mathrm{M}$ & 88.00 & Very good rock (I) \\
\hline 825 & & 43.00 & Fair rock (III) \\
\hline 850 & & 42.00 & \\
\hline 890 & M & 85.00 & Very good rock (I) \\
\hline 900 & $\mathrm{QMS}+\mathrm{M}$ & 42.00 & Fair rock (III) \\
\hline 907 & MS & 45.00 & \\
\hline 920 & QMS & 26.00 & Poor rock (IV) \\
\hline 927 & & 22.00 & \\
\hline 960 & & 87.00 & Very good rock (I) \\
\hline 975 & & 95.00 & \\
\hline 1008 & & 93.00 & \\
\hline
\end{tabular}




\section{Continued}

\begin{tabular}{|c|c|c|c|}
\hline 1040 & & 58.00 & Fair rock (III) \\
\hline 1060 & & 92.00 & Very good rock (I) \\
\hline 1075 & & 86.00 & \\
\hline 1085 & & 88.00 & \\
\hline 1100 & & 91.00 & \\
\hline 1110 & & 98.00 & \\
\hline 1120 & & 91.00 & \\
\hline 1130 & & 88.00 & \\
\hline 1150 & & 47.00 & Fair rock (III) \\
\hline 1175 & & 23.00 & Poor rock (IV) \\
\hline 1200 & & 26.00 & \\
\hline 1225 & CS & 23.00 & \\
\hline 1260 & $\mathrm{CS}+\mathrm{QMS}$ & 24.00 & \\
\hline 1280 & G_QMS + CS & 90.00 & Very good rock (I) \\
\hline 1290 & & 82.00 & \\
\hline 1315 & G_QMS & 90.00 & Very good rock (I) \\
\hline 1320 & G_QMS & 83.00 & \\
\hline 1385 & $\mathrm{M}+\mathrm{G} \_\mathrm{QMS}+\mathrm{CS}$ & 91.00 & \\
\hline 1425 & $\mathrm{QMS}+\mathrm{G}_{-} \mathrm{QMS}$ & 55.00 & Fair rock (III) \\
\hline 1445 & GMS + G_QMS & 56.00 & \\
\hline 1490 & $\mathrm{QMS}+\mathrm{G} \_\mathrm{QMS}+\mathrm{GMS}$ & 91.00 & Good rock (II) \\
\hline 1510 & QMS & 42.00 & Fair rock (III) \\
\hline 1525 & G_QMS + QMS & 47.00 & \\
\hline 1538 & G_QMS & 45.00 & \\
\hline 1549 & & 18.00 & Very poor rock $(\mathrm{V})$ \\
\hline 1555 & & 22.00 & Poor rock (IV) \\
\hline 1586 & & 13.00 & Very poor rock $(\mathrm{V})$ \\
\hline 1645 & QMS & 15.00 & \\
\hline 1653 & & 13.00 & \\
\hline 1660 & & 22.00 & Poor rock (IV) \\
\hline 1690 & & 13.00 & Very poor rock $(\mathrm{V})$ \\
\hline 1745 & GMS & 23.00 & Poor rock (IV) \\
\hline 1760 & & 16.00 & Very poor rock $(\mathrm{V})$ \\
\hline 1800 & & 24.00 & Poor rock (IV) \\
\hline 1808 & QMS & 42.00 & Fair rock (III) \\
\hline 1835 & & 45.00 & \\
\hline 1865 & & 44.00 & \\
\hline
\end{tabular}




\section{Continued}

\begin{tabular}{|c|c|c|c|}
\hline 1875 & & 45.00 & \\
\hline 1892 & & 43.00 & \\
\hline 1908 & & 42.00 & \\
\hline 1925 & & 56.00 & \\
\hline 1934 & & 46.00 & \\
\hline 1960 & GMS & 57.00 & \\
\hline 1980 & & 56.00 & \\
\hline 2000 & & 45.00 & \\
\hline 2025 & GMS + QMS & 48.00 & \\
\hline 2050 & QMS & 46.00 & \\
\hline 2065 & & 51.00 & \\
\hline 2086 & G_QMS + GMS & 44.00 & \\
\hline 2100 & GMS & 42.00 & \\
\hline 2125 & $\mathrm{QT}+\mathrm{MM}$ & 43.00 & \\
\hline 2141 & MM & 57.00 & \\
\hline 2150 & G_QMS & 50.00 & \\
\hline 2162 & & 57.00 & Fair rock (III) \\
\hline 2175 & M & 49.00 & \\
\hline 2186 & & 57.00 & \\
\hline 2200 & $\mathrm{QMS}+\mathrm{QT}$ & 46.00 & \\
\hline 2216 & & 47.00 & \\
\hline 2236 & QT & 58.00 & \\
\hline 2252 & & 47.00 & \\
\hline 2265 & & 53.00 & \\
\hline 2275 & & 47.00 & \\
\hline 2292 & & 55.00 & \\
\hline 2352 & & 46.00 & \\
\hline 2317 & & 58.00 & \\
\hline 2330 & & 36.00 & Poor rock (IV) \\
\hline 2347 & $\mathrm{QT}+\mathrm{QMS}$ & 42.00 & Fair rock (III) \\
\hline 2365 & QT & 45.00 & \\
\hline 2376 & $\mathrm{GMS}+\mathrm{M}$ & 39.00 & Poor rock (IV) \\
\hline 2393 & $\mathrm{M}+\mathrm{QMS}$ & 41.00 & Fair rock (III) \\
\hline 2400 & M & 47.00 & \\
\hline 2426 & & 41.00 & \\
\hline 2440 & & 42.00 & \\
\hline 2455 & & 47.00 & \\
\hline
\end{tabular}




\section{Continued}

\begin{tabular}{|c|c|c|c|}
\hline 2468 & & 75.00 & Good rock (II) \\
\hline 2475 & & 77.00 & \\
\hline 2503 & M & 84.00 & Very good rock (I) \\
\hline 2524 & & 85.00 & \\
\hline 2535 & & 82.00 & \\
\hline 2557 & & 57.00 & Fair rock (III) \\
\hline 2576 & & 74.00 & Good rock (II) \\
\hline 2587 & & 44.00 & Fair rock (III) \\
\hline 2594 & & 43.00 & \\
\hline 2603 & & 51.00 & \\
\hline 2610 & & 42.00 & \\
\hline 2624 & G_QMS + M & 45.00 & \\
\hline 2635 & G_QMS + QMS & 44.00 & \\
\hline 2652 & QMS & 26.00 & Poor rock (IV) \\
\hline 2673 & & 52.00 & Fair rock (III) \\
\hline 2682 & $\mathrm{QMS}+\mathrm{GMS}$ & 43.00 & \\
\hline 2689 & $\mathrm{GMS}+\mathrm{M}$ & 28.00 & Poor rock (IV) \\
\hline 2702 & & 43.00 & Fair rock (III) \\
\hline 2707 & GMS & 44.00 & \\
\hline 2714 & & 56.00 & \\
\hline 2720 & & 58.00 & \\
\hline 2727 & $\mathrm{GMS}+\mathrm{M}$ & 44.00 & \\
\hline 2733 & & 52.00 & \\
\hline 2740 & & 44.00 & \\
\hline 2746 & & 47.00 & \\
\hline 2751 & & 48.00 & \\
\hline 2760 & & 47.00 & \\
\hline 2773 & & 45.00 & \\
\hline 2782 & M & 48.00 & \\
\hline 2788 & $\mathrm{M}+\mathrm{GMS}$ & 45.00 & \\
\hline 2800 & GMS & 38.00 & Poor rock (IV) \\
\hline
\end{tabular}

GMS = Garnet Mica Schist, $\mathrm{M}=$ Marble, $\mathrm{MM}=$ Micaceous Marble, $\mathrm{QMS}=$ Quartz Mica Schist, G-QMS = Garnet bearing Quartz Mica Schist, $\mathrm{CS}=$ Calcareous Schist, $\mathrm{QT}=$ Quartzite, $\mathrm{MS}=$ Micaceous Schist.

\subsubsection{Quartz Mica Schist (QMS)}

The recommended Uniaxial Compressive Strength (UCS) of QMS is i.e. $80 \mathrm{MPa}$; $\mathrm{RQD}$ is $75 \%$, joints spacing ranging from $10-60 \mathrm{~cm}$, joints encountered are smooth-slicken sided and undulating-planar, persistence $3-10 \mathrm{~m}$ and joint apertures are $<1 \mathrm{~mm}$ in width with soft filling of materials. Fair to unfavorable 
orientation of discontinuities conditions were observed.

\subsubsection{Garnet Bearing Quartz Mica Schist (G-QMS)}

The average values UCS for G-QMS is $95 \mathrm{MPa}$ with average RQD 75\%, joints spacing ranges from $10-50 \mathrm{~cm}$, joints are undulating, planar and smooth, persistence of $3-10 \mathrm{~m}$ and joint apertures are $<1 \mathrm{~mm}$ in width with soft filling materials. Discontinuities attitude reveals fair to unfavorable tunnel drive conditions.

\subsubsection{Garnet Mica Schist (GMS) and Micaceous Schist (MS)}

The GMS has average UCS of $80 \mathrm{MPa}$ and RQD of 50\%, joint spacing range is 10 $-22 \mathrm{~cm}$, joint apertures $<1 \mathrm{~mm}$ in width with soft filling materials with undulating, planar, smooth \& slickensided joints, persistence of $0.3-3 \mathrm{~m}$.

\subsubsection{Marble (MB), Siliceous Marble (SM) and Micaceous Marble (MM)} The average UCS for these rocks is $80 \mathrm{MPa}$ with RQD of $60 \%$, joint apertures are $<1 \mathrm{~mm}-5 \mathrm{~mm}$ with non-softening fillings and clean also, rough, irregular, undulating \& smooth, $0.5-2.8 \mathrm{~m}$ of persistence and tunnel drive situation is favorable \& very favourable.

\subsubsection{Quartzite (QT)}

The average value of $145 \mathrm{MPa}$ were calculated as UCS strength for QMS with $\mathrm{RQD}$ is $47 \%$, joints spacing ranging from $10-20 \mathrm{~cm}$, joints encounter are irregular, smooth and undulating-planar, persistence of joints are $6-4 \mathrm{~m}$ and joint apertures are $<1 \mathrm{~mm}-3 \mathrm{~mm}$ in width with soft filling materials and few joints are clean. Very favorable to favorable orientation of discontinuities along tunnel route was observed.

\subsection{Estimated Support Recommendations}

The support estimation was calculated by both RMR and Q system. The Q-values and other required parameters (tunnel height and excavation support ratio) have been evaluated in the support estimate chart (Figure 2). The estimated support by Q system falls in category 1, 3, 4, 5, 6 and 7 which have support requirements of unsupported, systematic bolting, systematic bolting (shotcrete, $4-10 \mathrm{~cm}$ ), fiber reinforced shotcrete and bolting, $5-9 \mathrm{~cm}$, fiber reinforced shotcrete \& bolting, $9-12 \mathrm{~cm}$ and fiber reinforced shotcrete \& bolting, $12-15$ $\mathrm{cm}$ respectively as shown by red color square (Figure 2 ).

According to calculate values of RMR, rock masses characterized in following classes: Very good (I) and Fair (III) to Very poor (V). Based on support recommendation chart after Bieniawski, type and amount of support estimates are listed in the (Table 4).

\subsection{Correlation between Petrography and Rock Mass Parameters}

The detailed petrographic studies of rock samples revealed that tunnel majorly consist of five major rock types namely QMS, GMS, G-QMS, M and CS. The collected samples of QMS, M and G-QMS are fine to medium grained, compact 


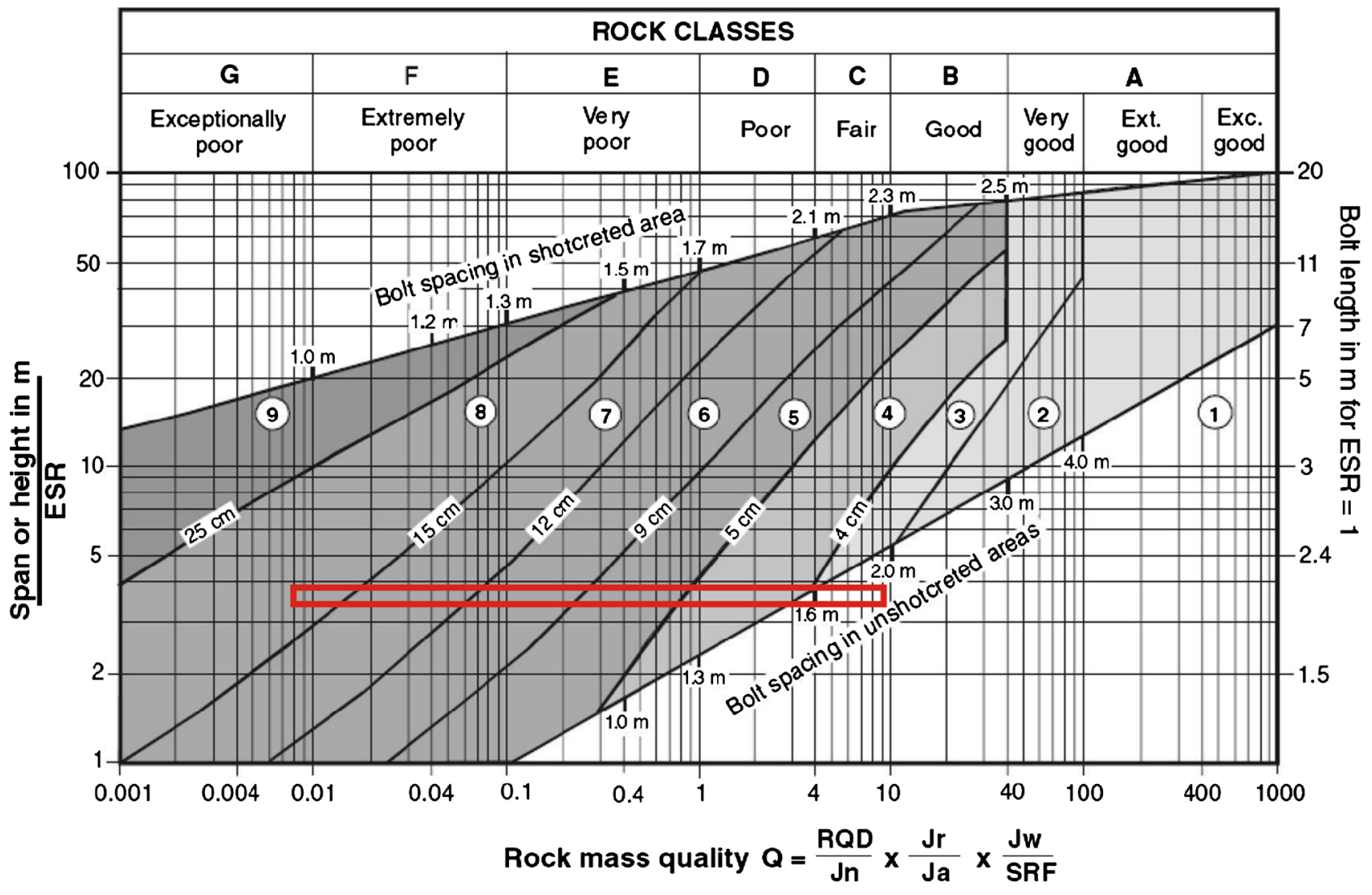

\section{REINFORCEMENT CATEGORIES:}
1) Unsupported
2) Spot bolting
3) Systematic bolting
4) Systematic bolting, (and unreinforced shotcrete, $4-10 \mathrm{~cm}$ )
5) Fibre reinforced shotcrete and bolting, $5-9 \mathrm{~cm}$
6) Fibre reinforced shotcrete and bolting, $9-12 \mathrm{~cm}$
7) Fibre reinforced shotcrete and bolting, $12-15 \mathrm{~cm}$
8) Fibre reinforced shotcrete, $>15 \mathrm{~cm}$,
reinforced ribs of shotcrete and bolting
9) Cast concrete lining

Figure 2. Estimated support categories based on the tunnelling quality index (Q). Red color represents the support categories for the studied tunnel (Modified after Barton [26]).

Table 4. Support recommendation based on RMR [2].

\begin{tabular}{|c|c|c|c|}
\hline \multirow[t]{2}{*}{ Rock Mass Class } & \multicolumn{3}{|c|}{ Support Recommendations } \\
\hline & Rock bolts & Shotcrete & Steel sets \\
\hline Class I & \multicolumn{3}{|c|}{ No support required except spot rock bolting } \\
\hline \multicolumn{4}{|l|}{ Very good rock } \\
\hline $\begin{array}{c}\text { Class II } \\
\text { Fair rock }\end{array}$ & $\begin{array}{c}3 \mathrm{~m} \text { long bolts in crown, spaced } 2.5 \mathrm{~m} \text { with } \\
\text { occasional wire mesh }\end{array}$ & $50 \mathrm{~mm}$ in crown where required & None \\
\hline $\begin{array}{l}\text { Class III } \\
\text { Poor rock }\end{array}$ & $\begin{array}{l}\text { Systematic bolt } 4 \mathrm{~m} \text { long, spaced } 1.5-2 \mathrm{~m} \text { in } \\
\text { crown and walls with wire mesh in crown }\end{array}$ & $\begin{array}{l}50-100 \mathrm{~mm} \text { in crown and } 30 \mathrm{~mm} \text { in } \\
\text { sides }\end{array}$ & None \\
\hline Class IV & $\begin{array}{l}\text { Systematic bolt } 4-5 \mathrm{~m} \text { long, spaced } 1-1.5 \mathrm{~m} \\
\text { in crown and walls with wire mesh }\end{array}$ & $\begin{array}{l}100-150 \mathrm{~mm} \text { in crown and } 100 \mathrm{~mm} \text { in } \\
\text { sides }\end{array}$ & $\begin{array}{l}\text { Light to medium ribs spaced } 1.5 \mathrm{~m} \\
\text { where required }\end{array}$ \\
\hline $\begin{array}{l}\text { Class } \mathrm{V} \\
\text { Very poor rock }\end{array}$ & $\begin{array}{l}\text { Systematic bolt } 5-6 \mathrm{~m} \text { long, spaced } 1-1.5 \mathrm{~m} \\
\text { in crown and walls with wire mesh, bolts invert }\end{array}$ & $\begin{array}{l}150-200 \mathrm{~mm} \text { in crown and } 150 \mathrm{~mm} \text { in } \\
\text { sides and } 50 \mathrm{~mm} \text { on the face }\end{array}$ & $\begin{array}{l}\text { Medium to heavy ribs spaced } 0.75 \\
\mathrm{~m} \text { with steel lagging and forepoling } \\
\text { if required. Close invert }\end{array}$ \\
\hline
\end{tabular}


Table 5. Average RQD, Q value and RMR rating with respect to main rock types.

\begin{tabular}{cccc}
\hline Rock types & Average RQD & Average Q-value & Average RMR rating \\
\hline GMS & $40 \%$ & 1.29 & 40 \\
CS & $41 \%$ & 0.43 & 29 \\
G-QMS & $57 \%$ & 3.91 & 62 \\
QMS & $43 \%$ & 1.14 & 38 \\
M & $45 \%$ & 3.08 & 47 \\
\hline
\end{tabular}

and are cross cut by few discontinuities having greater spacing. As a result, these rocks have greater average RQD, Q values, RMR ratings (Table 5). The remaining two rock types such as GMS and CS are relatively medium to coarse-grained with many closely spaced discontinuities. The average RQD, Q values and RMR ratings of these segments are comparatively low (Table 5).

\section{Conclusion}

The main objectives of the current research work are the rock mass categorization along proposed tunnel alignment using RMR and Q system and comparison of rock mass quality with mineralogical composition of rocks. The results were further materialized to predict and assess applicable rock reinforcement requirements for tunnel. Tunnel was divided into five major geological segments on the bases of rock type: quartz mica schist (QMS), garnet mica schist (GMS), garnet bearing quartz mica schist (G-QMS), calcareous schist (CS), marble (M). $Q$ values vary between 0.01 and 13.33 that depicted rock masse quality ranges from good to extremely poor in case of Q-system, while very good to very poor according to RMR. Geological segments comprised of following rock type quartz mica schist, marble and garnet bearing quartz mica schist having fine to medium-grained texture, compact and having large spacing. Therefore, these geological segments have greater average $R Q D, Q$ values, $R M R$ ratings as compared to garnet mica schist and calcareous schist.

\section{Conflicts of Interest}

The authors declare no conflicts of interest regarding the publication of this paper.

\section{References}

[1] Bell, F.G. (1995) The Significance of Engineering Geology to Construction. Geological Society, London, Engineering Geology Special Publications, 10, 3-29. https://doi.org/10.1144/GSL.ENG.1995.010.01.01

[2] Bieniawski, Z.T. (1973) Engineering Classification of Jointed Rock Masses. Transaction of the South African Institution of Civil Engineers, 15, 335-344.

[3] Deere, D.U. and Miller, D.W. (1967) The Rock Quality Designation (RQD) Index in Practice, Classification Systems for Engineering Purposes. ASTM STP, American Society for Testing and Materials, Philadelphia, PA, 91-101. 
https://doi.org/10.1520/STP48465S

[4] Wickham, G.E., Tiedemann, H.R. and Skinner, E.H. (1972) Support Determination Based on Geologic Predictions. In: Lane, K.S.A.G., Ed., North American Rapid EXcavation and Tunneling Conference, Society of Mining Engineers of the American Institute of Mining, Metallurgical and Petroleum Engineers, Chicago, IL, New York, 43-64.

[5] Bieniawski, Z.T. (1989) Engineering Rock Mass Classifications. John Wiley \& Sons, New York, 251.

[6] Cosar, S. (2004) Application of Rock Mass Classification Systems for Future Support Design of the DM Tunnel Near Alanya. Middle East Technical University, Ankara, Turkey, 217.

[7] Abu-Zeid, N. and Vuillermin, F. (1997) Geochemical Characterisation of the Outcropping Rock Masses of the Immediate Vicinity of Wadi El-Kaffrein Dam Site (Jordan). Bulletin of the International Association of Engineering Geology, 55, 3-17. https://doi.org/10.1007/BF02635404

[8] El-Naqa, A. (2001) Application of RMR Q Geomechanical Classification Systems along the Proposed Mujib Tunnel Route, Central Jordan. Bulletin of Engineering Geology and the Environment, 60, 257-269.

https://doi.org/10.1007/s100640100112

[9] Barton, N. (2002) Some New Q-Value Correlations to Assist in Site Characterization and Tunnel Design. International Journal of Rock Mechanics and Mining Sciences, 39, 185-216. https://doi.org/10.1016/S1365-1609(02)00011-4

[10] Choi, S.Y. and Park, H.D. (2002) Comparison among Different Criteria of RMR and Q-System for Rock Mass Classification for Tunneling in Korea. Tunneling and Underground Space Technology, 17, 391-401. https://doi.org/10.1016/S0886-7798(02)00063-9

[11] Ramamurthy, T. (2004) A Geo-Engineering Classification for Rocks and Rock Masses. International Journal of Rock Mechanics and Mining Sciences, 41, 89-101. https://doi.org/10.1016/S1365-1609(03)00078-9

[12] Hoek, E. and Diederichs, M.S. (2006) Empirical Estimation of Rock Mass Modulus. International Journal of Rock Mechanics and Mining Sciences, 43, 203-215. https://doi.org/10.1016/j.ijrmms.2005.06.005

[13] Naithani, A.K., Bhatt, A.K. and Murthy, K.S.K. (2009) Geological and Geotechnical Investigations of Loharinag-Pala Hydroelectric Project, Garhwal Himalaya, Uttarakhand. Journal of the Geological Society of India, 73, 821-836. https://doi.org/10.1007/s12594-009-0066-0

[14] Ozsan, A., Hakan, B., Serdar, Y. and Ozkan, C. (2009) Engineering Geological Evaluation and Preliminary Support Design for the Metro Extension Tunnel, Ankara, Turkey. Bulletin of Engineering Geology and the Environment, 68, 397-408. https://doi.org/10.1007/s10064-009-0192-3

[15] Gupta, M.C., Singh, B.K. and Singh, K.N. (2011) Engineering Geological Rock Mass Classification of Punasa Tunnel Site, Khandwa District, Madhya Pradesh. Journal of the Geological Society of India, 77, 269-272. https://doi.org/10.1007/s12594-011-0034-3

[16] Gurocak, Z. (2011) Analyses of Stability and Support Design for a Diversion Tunnel at the Kapikaya Dam Site, Turkey. Bulletin of Engineering Geology and the Environment, 70, 41-52. https://doi.org/10.1007/s10064-009-0258-2

[17] Mondal, M.E.A., Siddique, T., Alam, M., and Alam, M.M. (2016) Rock Mass Rating and Kinematic Analysis for Slope Stability Investigation of Utari Dam, Lalitpur Dis- 
trict, Uttar Pradesh. Journal Geological Society of India, 87, 463-468. https://doi.org/10.1007/s12594-016-0414-9

[18] Akram, M.S., Mirza, K., Zeeshan, M. and Jabbar, M.A. (2018) Assessment of Rock Mass Quality and Deformation Modulus by Empirical Methods along Kandiah River, KPK, Pakistan. Open Journal of Geology, 8, 947-964. https://doi.org/10.4236/ojg.2018.810057

[19] Akram, M.S. and Zeeshan, M. (2018) Rock Mass Characterization and Support Assessment along Power Tunnel of Hydropower in Kohistan Area, KPK, Pakistan. Journal of the Geological Society of India, 91, 221-226. https://doi.org/10.1007/s12594-018-0839-4

[20] Akram, M. S., Zeeshan, M., Mirza K., Noor, A. and Ahmed, L. (2019) Slope Stability Analyses Using Classification Systems and Numerical Methods: Case Study from Lower Dir, Khyber Pakhtunkhwa, Pakistan. Himalayan Geology, 40, 67-77.

[21] Akram, M.S., Mirza K., Zeeshan, M., Ali, M. and Ahmed, L. (2018) Geotechnical Investigation and Prediction of Rock Burst, Squeezing with Remediation Design by Numerical Analyses along Headrace Tunnel in Swat Valley, Khyber Pakhtunkhwa, Pakistan. Open Journal of Geology, 8, 965-986.

https://doi.org/10.4236/ojg.2018.810058

[22] Calkins, J.A., Offield, T.W., Abdullah, S.K.M. and Tayyab, A.S. (1975) Geology of the Southern Himalaya in Hazara, Pakistan and Adjacent Areas. U.S Geological Survey Professional Paper 716C, 29. https://doi.org/10.3133/pp716C

[23] Treloar, P.J., Broughten, R.D., Coward, M.P., Williams, M.P. and Windley, B.F. (1989) Deformation, Metamorphism and Imbrication of the Indian Plate South of MMT, North Pakistan. Journal of Metamorphic Geology, 7, 111-127. https://doi.org/10.1111/j.1525-1314.1989.tb00578.x

[24] Le Fort, P., Debon, F. and Sonet, J. (1980) The "Lesser Himalayan" Cordierite Granite Belt, Typology and Age of the Pluton of Mansehra, Pakistan. Geological Bulletin, 13, 51-62.

[25] Latif, M.A. (1970) Explanatory Notes on the Geology of Southern Hazara to Accompany the Revised Geological Map. Wein Jahrbuch der Geologischen Bundesanstalt, 15, 5-20.

[26] Barton, N., Lien, R. and Lunde, J. (1974) Engineering Classification of Rock Masses for the Design of Rock Support. Rock Mechanics, 6, 189-236. https://doi.org/10.1007/BF01239496

[27] Palmstrom, A. (1996) RMi-A System for Characterizing Rock Mass Strength for Use in Rock Engineering. Journal of Rock Mechanics and Tunneling Technology, 1, 69-108.

[28] Hoek, E. and Brown, E.T. (1997) Practical Estimates of Rock Mass Strength. International Journal of Rock Mechanics \& Mining Sciences, 34, 1165-1186. https://doi.org/10.1016/S1365-1609(97)80069-X

[29] Palmstrom, A. (2001) Measurement and Characterization of Rock Mass Jointing. In: Sharma, V.M. and Saxena, K.R., Eds., In-Situ Characterization of Rocks, A.A. Balkema Publishers, London, 97.

[30] International Society for Rock Mechanics (ISRM) (1990) Basic Geotechnical Description of Rock Masses. International Journal of Rock Mechanics and Mining Sciences and Geomechanics Abstract, 18, 85-110. 